\title{
Admins, Mods, and Benevolent Dictators for Life: The Implicit Feudalism of Online Communities
}

\author{
Nathan Schneider \\ University of Colorado Boulder \\ New Media 83 Society (2021) \\ https://doi.org/10.1177/1461444820986553
}

\begin{abstract}
Online platforms train users to interact with each other through certain widespread interface designs. This article argues that an 'implicit feudalism' informs the available options for community management on the dominant platforms for online communities. It is a pattern that grants user-administrators absolutist reign over their fiefdoms, with competition among them as the primary mechanism for quality control, typically under rules set by platform companies. Implicit feudalism emerged from technical conditions dating to early online networks. In light of alternative management mechanisms with more democratic features, it becomes all the more clear that implicit feudalism is not a necessary condition.
\end{abstract}

The author is grateful for constructive feedback on earlier drafts from Kevin Driscoll, Seth Frey, Benjamin Mako Hill, Alyssa Rosenzweig, Edward Saperia, and anonymous reviewers.

\section{Introduction}

In contrast to field-forming work that heralded online communities as democratic vanguards (Benkler, 2006; Castells, 2015), analyses of anti-democratic tendencies lurking in the thrall of major Internet platforms have grown more common (Noble, 2018; Tufekci, 2017; Zuboff, 2019). The trouble for democracy considered here is more intimate than the treatments of how platforms mediate users' relationships to macro-level institutions and discourses. This article considers how those platforms train users to interact 
with each other through certain widespread interface designs. I argue that an 'implicit feudalism' informs the available options for community management on the dominant platforms for online communities. It is a pattern that grants user-administrators absolutist reign over their fiefdoms, with competition among them as the primary mechanism for quality control, typically under rules set by platform companies. These practices emerged from technical conditions dating to early social platforms. In light of alternative management mechanisms with more democratic features, it becomes all the more clear that implicit feudalism is not a necessary condition.

By implicit I mean that while platforms may not explicitly proclaim or seek to practice feudal ideology - to the contrary, many claim democratic ideals - a feudalism lurks latent in the available tools that guide and limit user behavior. I do not use feudalism in a historically precise sense, as there is much to distinguish online communities from the medieval European regime of land tenancy and its lord-vassal relations. Rather, I use the word metaphorically to describe concurrent communities across a network, each subject to a power structure that is apparently absolute and unalterable by those who lack such power. I do not here dwell on ways in which the digital economy appears to be fostering a new feudalism of wealth inequality, although governance and economy frequently intermingle. Nor do I necessarily attach to feudalism the pejorative connotations the word often carries. Many of the feudal practices considered here have been sensible and efficient; they may be especially appropriate at certain stages of a community's life-cycle, such as early on or at key points of transition. Implicit feudalism places unnecessary limits on the possibilities available to communities, but even in the presence of a wider range of options a feudal structure may at times be the best choice.

With the language of democracy, I similarly run the risk of caricature. I mean simply a broad family of governance systems that seek to inscribe authority within commonly agreed-upon rules and that hold the persons in authority accountable to those they govern (Diamond, 2004). A general hallmark of accountability is that those subject to an authority have the capacity to transfer the authority to someone else. The mechanisms necessary for many basic democratic processes are missing from the design patterns of implicit feudalism.

Democratic practices can emerge among feudal technologies, such as when moderators feel rhetorical or social pressure to respect the values of community members in how they exert their otherwise absolute authority. Feudal power can thereby exhibit forms of accountability that Stasavage (2020) calls 
"early democracy." Communities may repurpose features like emjois and polls to carry out governing functions. But under implicit feudalism, inclusive governance tends to require ad hoc adaptations of available feature-sets, against the grain of user-interface nudges. Some empirical studies have concluded that non-democratic regimes are a natural or at least likely outcome in online communities, following the prediction of sociologist Robert Michels's 1915 'iron law of oligarchy' (Frey and Sumner, 2019; Shaw and Hill, 2014). Yet upon examining what the available tools allow, the observed oligarchic outcomes begin to seem pre-ordained. Implicit feudalism has forestalled social and political questions of how community governance might otherwise occur.

I have been tempted to consider implicit feudalism a species of communicative affordance as Schrock (2015) defines it - 'an interaction between subjective perceptions of utility and objective qualities of the technology that alter communicative practices or habits' - or, in Nagy and Neff (2015)'s evocative phrase, an imagined affordance. Yet by the standards of Evans et al. (2017), implicit feudalism falls short of affordance-status, since social network developers do not advertise implicit feudalism as a feature; rather, it lurks in what the advertised affordances lack. To clarify the concept, this study adopts a media-archaeology approach, probing artifacts of the past in the infrastructure of the present (Huhtamo and Parikka, 2011; Malloy, 2016) specifically, the ways in which technical contexts of early online communities organized, and still organize, the realm of the possible. A close examination of historical documents and of the machines that accompanied them reveals how implicit feudalism formed in the negative space among platform features. Feudal governance catered to the technical circumstances of the platforms, as well as to the offline legal forms of ownership and control over their hardware. Corporations found benefits in encouraging centralized control among user communities. We could call implicit feudalism an 'imagined sufficiency' - a willingness to accept, and a failure to question, systems with impoverished feature-sets.

Familiar mechanisms of offline organizational life have been absent from online communities. From early bulletin-board systems to today's Facebook Groups, one finds few provisions for such democratic practices as elected boards, term limits, juries, or referendums. Imagined sufficiency has tended to envelop community participants, the admins among them, and platform designers alike. I hope that this study aids in unraveling such acceptance, much as the '\#darkpatterns' campaign among user-experience professionals seeks to dissuade peers from techniques that 'trick users into doing things' against 
or without their will (Fansher et al., 2018). Noticing implicit feudalism is surely the first step in making it less ubiquitous.

My analysis also adopts economist Albert O. Hirschman (1970)'s classic distinction between the signals of 'exit' and 'voice' in organizational life. Exit is the capacity to depart, such as by quitting a job or shopping with a competitor; voice is the capacity to make change from within, such as by campaigning one's city council for a local policy change. Although online community platforms seem and claim to facilitate the expression of voice, most users' voices do not hold instrumental power; users can speak out, but they must wait for admins to act on their complaints. If one doesn't like how a community is being run, one's primary recourse is exit - to chose another community or create one's own in an open market. Yet, as in other kinds of markets, the social costs of exit can be higher than they appear from a purely technical standpoint.

This article begins with a genealogy of implicit feudalism in online communities, chronicling its emergence among particular network structures before and during the early Internet. These appear to feed directly into more recent platforms for online communities, from peer-production tools to social media. I then summarize the feudal model and consider ways in which platforms might introduce more diverse forms of user governance outside the habits of implicit feudalism.

\section{Paradigm communities}

Implicit feudalism has its origins among the earliest online communities, due to technological circumstances that accorded well with feudal practices. The case studies that follow examine platforms and networks that have changed over time. I draw on historical examples but, when in the present tense, refer to the state of the art as of this writing. The choices of examples are selective - genealogically significant, I argue, but inevitably incomplete. Early social news platforms, video streaming platforms, question-and-answer platforms, gaming platforms, and productivity platforms are conspicuously absent, as is the parallel story of platforms behind China's 'Great Firewall.' I leave it to experts in those and other contexts to determine whether they recognize continuity with the implicit feudalism described here. 


\section{Progenitors: BBS, Usenet, and e-mail lists}

Predating the Internet, public bulletin board systems first appeared in the late 1970s (Christensen and Seuss, 1978; Malloy, 2016; Rheingold, 1993), offering computer hobbyists outside of academia and military-funded research centers their first experience of digitally mediated community. BBSes typically resided on a single user's computer at that user's home, running one or another variant of specialized, customizable BBS software (Figure 1). Other users' computers could log in through a phone line, post messages and files, and download content others had posted. The user who hosted a BBS became known as a 'sysop,' short for 'system operator.' Interviewees in BBS: The Documentary (Scott, 2005) testify to the intimacy of the sysop experience. One sysop describes lying in bed and being able to infer what users were doing on the BBS from the sounds of the computer on the other side of the room (episode 2, 11:30). Many sysops thus regarded users as guests in their homes, resulting in both generous and domineering behaviors. 'This bulletin board is in my house,' a sysop might declare, 'I will not have any swear words on it!' (17:20)

With hospitality came power. As a sysop, says one informant, 'You could do whatever you wanted' (6:35). Says another, 'At the end of the day, it is the sysop who is the ultimate judge, jury, and executioner' (16:40); after all, the sysop could say, 'If you don't like it, get off my computer, get out of my phone lines!' Mirroring a home computer's access control system, all rights emanated from the sysop.

Kevin Driscoll (2017) recounts how sysops found themselves becoming not just hosts but lawgivers through their unique relationship to the system:

In moments of conflict, sysops became mediators. They were the makers and enforcers of social policy. Ultimately, the sysop possessed a form of total authority because they lived under the same roof as the host PC. In a moment of frustration, the sysop could always pull the plug and shut down the whole system.

While a sysop's absolute power stemmed from the power to terminate the community, users had power of their own stemming from the option to exit- to leave one BBS for another:

if a user or group of users found themselves in an unresolvable conflict with a sysop, they were always free to depart and create their own system. The freedom for users to leave the system 


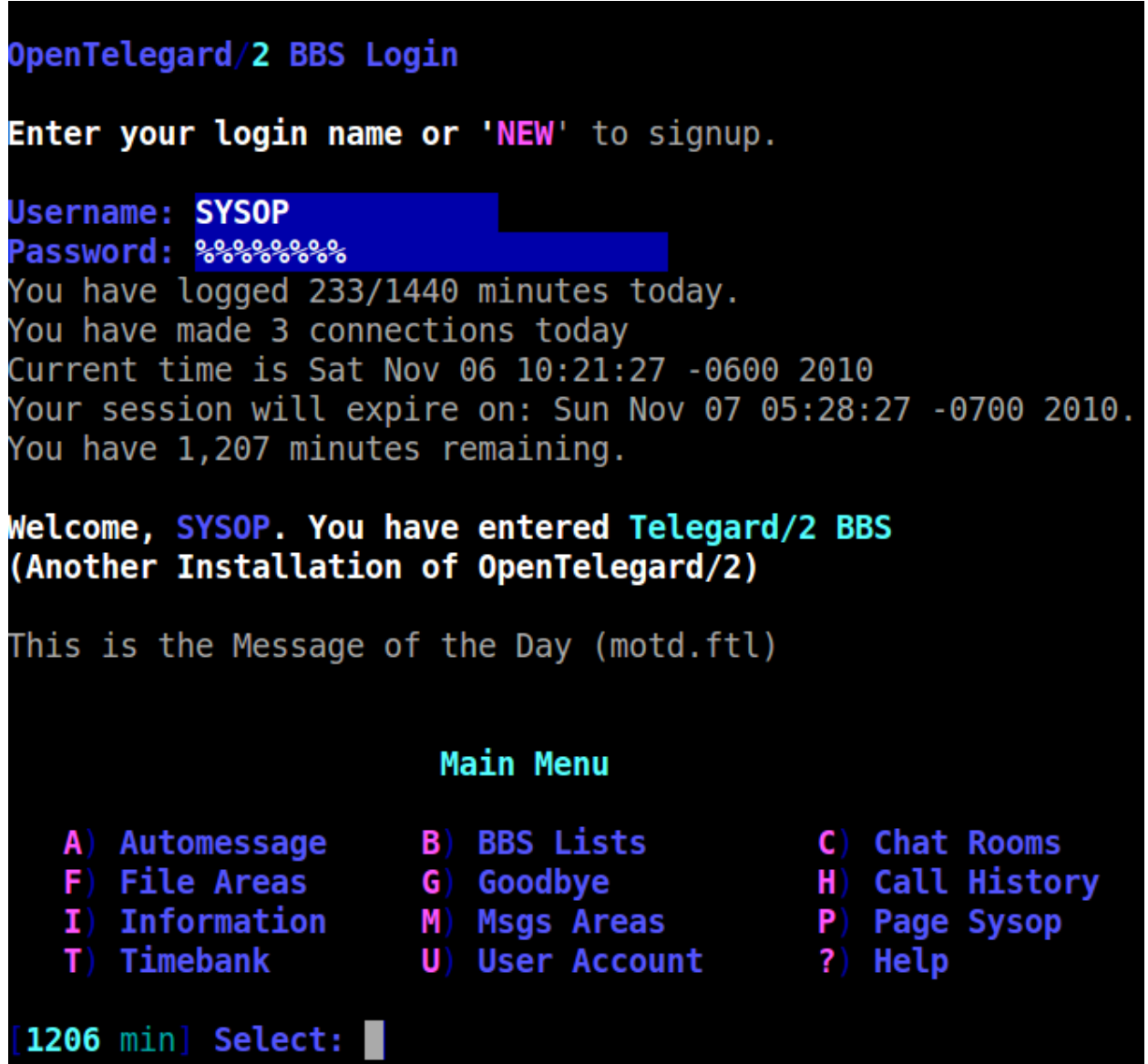

Figure 1: Screenshot of the OpenTelegard BBS software (https://en.wikipedia.org/wiki/File:20101106_opentelegard_login_screenshot.png). 
created a check on sysops' power and created a sense of mutual accountability within the community.

The first specification for a BBS (Christensen and Seuss, 1978) describes the system operator (not yet a sysop) as a technical functionary, performing maintenance on the machine and using special message-deletion privileges in cases of user carelessness. But before long, Driscoll explains, the notunder-my-roof spirit infused the feature-set of BBS software, granting sysops fine-grained authority to sanction and censor users. There were also more democratic options available, such as the votemgr program for the FidoNet BBS network (Castillo, 1991) or the OneNet network's constitution and board structure (OneNet, 1999). But the ownership of the non-virtual hardware bled into virtual feudalism. After the later LambdaMOO online community experimented with democracy and then reverted to feudal governance, one of its 'wizards' explained the reason in terms of offline jurisdiction:

so long as the MOO is located on a single RL [real-life] machine at a single RL site subject to RL laws and liabilities, there will be those deemed responsible for the use of that hardware. (Ludlow, 2001: 324)

In the BBS lies the paradigm case of implicit feudalism, a pattern understandable and perhaps inevitable for communities physically residing in a particular member's home. Later manifestations of this pattern grow progressively less inevitable.

In 1980, another approach to networked community appeared in the form of Usenet (Malloy, 2016; Spencer and Lawrence, 1998). Like a BBS, it was a forum for asynchronous content-posting. But rather than residing in a sysop's home, Usenet distributed its 'newsgroups' across multiple, inter-operable servers. As a more public commons, its governance could not solely resort to a BBS-like smattering of residential fiefdoms. In the 'Great Renaming' of 1986, the system's major forums were restructured and placed under the authority of an organization eventually known as the Big 8 (Big-8.org, 2013). The Big 8 (still) governs key sections of Usenet through a Management Board that has the power to create and remove newsgroups. The Big 8 board is self-perpetuating, meaning that current members choose future members. Newsgroup moderators are chosen by previous moderators according to processes specified in a Big 8-approved group charter or, if there is a break in the line of succession, by the Big 8 board. Once chosen, moderators' power is much like that of a BBS sysop. 
Usenet's governance regime was robust enough to foster a popular set of online communities that, more than the home-bound BBSes, served as a virtual public square both before and after the rise of the Internet (Kollock and Smith, 1996). The circumstances of operating on a shared network, and taking up shared server space, required governance mechanisms capable of at least some collective decision making. That layer at least somewhat mitigated the implicit feudalism in Usenet's groups.

Among people with access to the ARPANET and the early Internet, email was the 'killer app' - the use-case that made the technology truly useful (Hafner and Lyon, 1996). As email became a medium for communities, it became another site of implicit feudalism. By the mid-1980s, the emaildiscussion software ListServ began replacing Usenet on university systems (Hyman, 2003). Other email-list programs, such as Sympa and Mailman, emerged later in the 1990s. Google Groups, both an email-list platform and a gateway to Usenet, appeared in 2001.

Email lists can take many forms, ranging from announcement lists to moderated or unmoderated discussion lists. But implicit feudalism infuses every major email-list system. Lists have particular admins or moderators, beginning with the list founders and followed by whomever they appoint. Organizations such as universities may provide meta-governance over the lists they allow on their servers, much as the Big 8 board did for Usenet. But beyond that, email-list software grants list admins full authority over such matters as list membership, posting rights, and documentation about the list's purpose and policies.

The implicit power structure inculcates cultural norms. According to a widely circulated post on a computer-security email list, 'Mailing lists should be run as an autocracy with the admins/owners as the rulers and the charter as the law' (Caines, 2013). Further, the poster regards the notion of 'selfmoderation' among users as an 'ill-considered and badly implemented mockery of a democratic process [or witch hunt, depending on your perspective].' By this account, the autocracy in a well-developed list may have a constitutionlike charter that lays out certain rights and responsibilities. But any such feature would be extraneous to the list software and must be implemented voluntarily by moderators. Feudalism is the presumed logic of the underlying systems. 


\section{Creators: Free/open-source software and Wikipedia}

An often-cited source of democratic promise in Internet culture is the family of practices that Benkler (2006) refers to as 'commons-based peer production.' The success of such projects as the Linux kernel and Wikipedia indicate that peer production is capable of producing highly scalable, successful infrastructure. Yet disappointments recur as decentralized peer-production projects - from the World Wide Web itself to Bitcoin - repeatedly produce centralized outcomes (Schneider, 2019). The disappointments might be more predictable were we to take seriously the implicit designs inscribed into these modes of production.

The Linux governance model centers around founder Linus Torvalds, who is popularly referred to as the project's 'benevolent dictator for life,' or BDFL (Federman, 2006; Laffan, 2012). But perhaps more influential than the Linux model has been its underlying software: Git, the version-control system that Torvalds developed in 2005 to manage the development of Linux, and which became a standard tool for free/open-source software projects.

The core Git software is itself remarkably pliable, designed to enable distributed software development, with multiple developers working on their particular modified versions of the code-base. No one version is intrinsically canonical. Git itself provides no mechanisms for governance. Although developers must eventually adopt one canonical code-base for release, Git leaves a power vacuum. Torvalds filled it for Linux with his BDFL status - quite simply, he decides which version is canonical and which community contributions it includes. Linux and many other projects employ email discussion lists for the discussion and decision-making that Git itself doesn't support. BDFL or not, the implicit feudalism of email lists was initially the go-to answer to Git's power vacuum. Whoever controls the list controls the process.

In the years following its release, Git has become most widely used through hosted platforms, particularly GitHub, a commercial service that Microsoft purchased in 2018 for $\$ 7.5$ billion. GitHub fills the Git power vacuum with an access and permissions system that identifies a single 'owner' and multiple 'collaborator' roles in a given project. The founder of a project begins as its owner and remains so until they assign someone else to that role. External users can also 'fork' a copy of the project, edit it, and either submit their changes back to the original as a 'pull request' or attempt to manage a competing version on an ongoing basis. As a centralized platform, unlike Git itself, GitHub establishes a canonical code-base for any given project, 
managed by its permissions system. GitHub inserts a feudal governance model to its cloud-based system for public-facing projects. The company also adds to Git a mechanism for contributor voice through its 'Issue' discussion system.

Magna Cartas have begun appearing as a check against Git's feudalism. Widespread abuses of power-for instance, Linus Torvalds' notoriously rude treatment of developers - helped give rise to codes of conduct for software projects, which seek to limit the scope of acceptable behavior and specify the responsibilities of admins (Tourani et al., 2017). Linux itself has adopted the popular Contributor Covenant, and GitHub encourages project owners to adopt a code of conduct as well. A code of conduct on the platform, however, depends on the project owner's willingness to abide by and enforce it.

Less feudal approaches are evidently possible. The Debian Project, which produces an important Linux-based operating system, self-governs through a liberal-democratic process (Coleman, 2013; Fish et al., 2011). Its Debian Constitution specifies various mechanisms, including the election of a Project Leader by Debian's developers. Skilled developers join the organization through a detailed and meritocratic on-boarding process. But in its formal republicanism, Debian has been mainly an outlier. Much free/open-source software development is centered around a particular benevolent dictator (Cosentino et al., 2014), an outcome not specifically dictated by Git but nudged by the implicit feudalism in the most conventional means of filling its power vacuums - email lists and centralized platforms like GitHub. Democratic structures appear only occasionally, usually among more developed software communities such as Debian and the Apache Software Foundation, whose developer-members elect their nonprofit organization's board. Nonprofit legal entities may be more welcoming to democratic communities than for-profit companies.

Likewise, the online encyclopedia Wikipedia operates through a sophisticated democracy among active volunteers. Wikipedia also possesses a widely acknowledged benevolent dictator in the person of founder Jimmy Wales, who prefers the metaphor of 'constitutional monarch' (Reagle, 2010, ch. 6) and oversees a complex of tiered roles, open participation, and electioneering from a "founder's seat" on the Wikimedia Foundation board (Jemielniak, 2014; Kostakis, 2010). His powers have diminished after several cases of overreach. Any user, in principle, can ascend the ranks of influence and position-holding such roles as 'administrator,' 'steward,' and 'bureaucrat' (Figure 2). Users are elected to these roles by their peers. The outlier on 
English Wikipedia is the role known as 'Jimmy Wales.' According to the website's documentation,

Jimmy Wales holds a special role in the governance of the English Wikipedia, due to the central and vital stake he had in its founding. This authority is used on an ad hoc basis, when other decision-making structures are inadequate or have failed in a particular situation. (Wikipedia, 2020)

Although one of the 'Five Pillars' of Wikipedia is that 'Wikipedia has no firm rules,' contributors have assembled a formidable assortment of policies on dozens of subjects. Aside from some external email and chat forums, most of the platform's governance occurs on the initially blank, editable pages of Wikipedia itself, formatted according to certain norms. It is a remarkable instance of 'eating your own dogfood' - an organization using its product in the process of making that same product.

The free/open-source software underlying Wikipedia, MediaWiki, is in principle available for others seeking to replicate the famous encyclopedia's success and governance model. However, without the long-cultivated norms around the use of 'Talk' pages and a complex system of permissions and roles, the software itself offers little in the way of democratic nudging. Like most Webbased platforms, a new deployment of MediaWiki grants privileges solely and completely to its administrator. It is not therefore surprising that, as Shaw and Hill (2014) found in a study of 683 MediaWiki-based deployments on the commercial platform Wikia, most use-cases tend toward oligarchical governance. Without Wikipedia's deliberate cultivation of democratic and bureaucratic process, the software facilitates a long tail of feudalism. Governance on Wikipedia itself has drifted toward less inclusivity and dynamism over time (Halfaker et al., 2013).

In principle, the power vacuums that software designs leave open should allow for diversity and a genuine meritocracy. But as feminist activist and scholar Jo Freeman (1972) famously observed, a 'tyranny of structurelessness' frequently arises - one in which the absence of an explicit hierarchy in a system results in an hidden, difficult-to-alter hierarchy imported from external social forces. The notion that 'anyone' can contribute to and even co-govern a free/open-source project to the extent that they are self-motivating and competent - a notion sometimes referred to as 'do-ocracy' (Zacchiroli, 2011) can fail to recognize that not everyone is equally equipped with the free time and other resources to participate. Power vacuums can produce the most entrenched hierarchies. 


\section{User access levels}

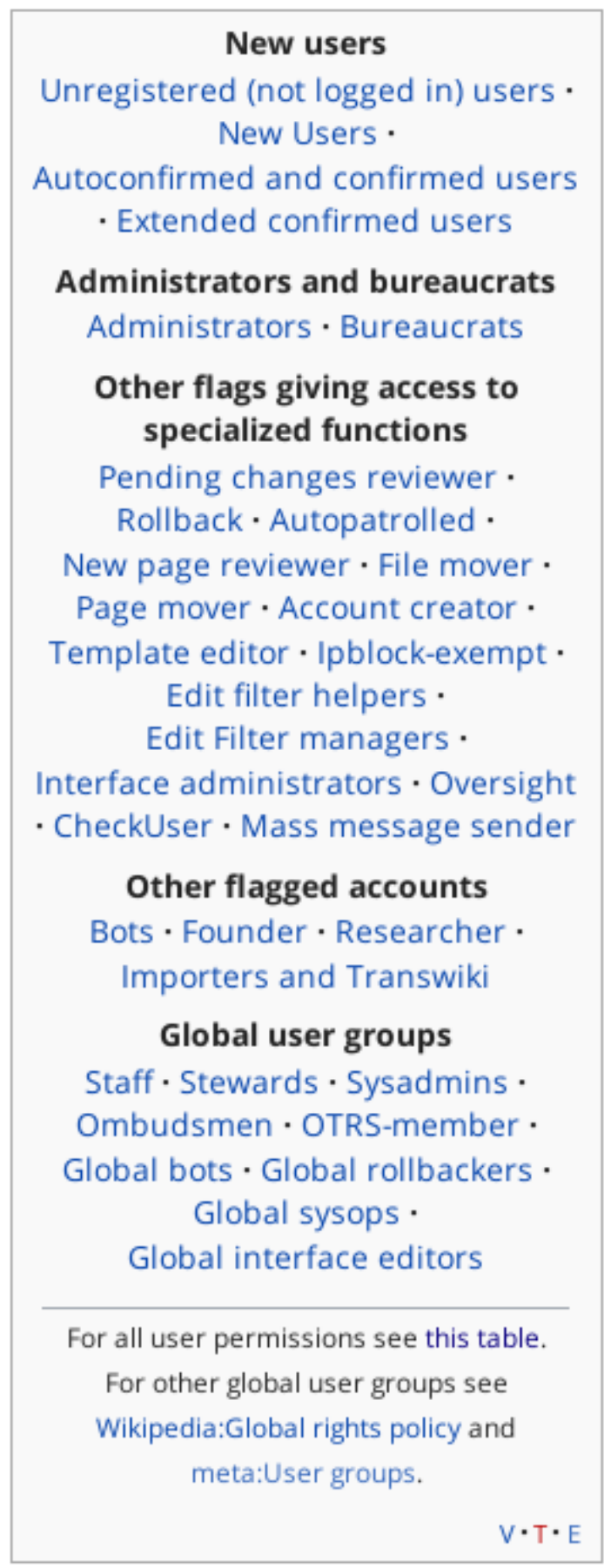

Figure 2: List of Wikipedia access levels (screenshot by author at https://en.wikipedia.org/wiki/Wikipedia:User_access_levels). 


\section{Platforms: Facebook, Reddit, and chat}

The rise of platforms controlled by globe-spanning corporations presented an opportunity for departing from the implicit feudalism of earlier systems. As Facebook's public-relations apparatus was beginning to come to terms with the platform's contested role in the 2016 US election, CEO Mark Zuckerberg issued a lengthy essay called 'Building Global Community' (2017). In it, he indicated a turn toward emphasizing 'meaningful groups' over the user-curated political news that was making the platform notorious. Recognizing the limits of the company's regulatory capacity, he mused about the opportunity to 'explore examples of how community governance might work at scale.'

Facebook, the world's largest private social-media network with over 2 billion active users (Clement, 2019), has had a Groups feature since 2005, the year after the website first appeared. 2005 also marked the beginning of Reddit, a social-news platform that by 2008 came to be organized around usercreated and user-governed groups known as Subreddits. Reddit's active-user population is about a tenth that of Facebook's, which still places it among the top ten US networks. In many respects, the two platforms are quite different; Facebook emphasizes users' 'real names' and mutual connections, while Reddit tends to rely on individualized, pseudonymous identities marked with reputation-based 'Karma.' Yet both adopt and further advance the pattern of implicit feudalism inherited from earlier networks like BBSes and email lists, despite lacking many of their predecessors' technological constraints.

A Facebook Group is not a BBS in one's home; it inhabits a corporation's platform. A Subreddit doesn't consume the server and bandwidth resources of its administrator, like a mailing list, only that of Reddit itself. It is no longer so obvious that the founder of a community should have dictatorial say over it. The norms and design elements of implicit feudalism are no longer a matter of technical necessity. But they became a business model.

One of the early commercial platforms for online communities, America Online, appointed 'community leaders' to moderate its chat rooms and message boards in exchange for reduced cost of access - a program that drew scrutiny from the Department of Labor as under-compensated work (Margonelli, 1999). Since then, platforms have avoided such gray-area compensation and instead relied on the allure of implicit feudalism to incentivize the labor of community management. User authority has become a means of 
uncompensated outsourcing.

One apparently democratic feature that appears in both Facebook and Reddit is the ability for non-moderator users to evaluate fellow users' postings - on Facebook, with the 'Like' button and its various affective sub-options, and on Reddit with 'upvotes' and 'downvotes.' Although the precise mechanics are not public knowledge, these tools allow users to mutually decide which content is more worth each other's attention, and thus which should rise to the top of the group's feed. The platforms also allow users to add comments, which have amplifying effects as well. However, the most definitive powers of amplification (elevating messages to the top of a group's feed) and sanction (ejecting posts and users) are reserved for those with administrative roles, who gain their roles by appointment and succession deriving from the group's founder. Seering et al. (2019) noticed, through interviews with admins on both platforms, that they rarely consult with non-admins on decisions about how to use these powers. Ordinary users' evaluative tools thus seem to operate as assists on behalf of admins (Leavitt and Robinson, 2017) and proprietary algorithms more than they are a means of shared governance. The strongest form of sanction that ordinary users have available remains that of exit - to leave a given Facebook Group or Subreddit for another, or to start a new one.

Facebook and Reddit implement advancements in implicit feudalism over earlier paradigms. For instance, rather than merely offering blank text fields for rule-making, as in MediaWiki and GitHub, these platforms have developed structured rule-making interfaces for group admins (Figures 3 and 4). Artificial intelligence tools, such as Facebook's 'false news' detector and Reddit's programmable AutoModerator (Jhaver et al., 2019), offer to streamline the labor of moderating content. Analytics dashboards present admins with detailed reports on the activity of their groups, in effect gamifying the admin role toward maximizing user usage (Figure 5). Such powers add to the panopticism and potency of implicit feudalism's repertoire.

Feudal community governance also exhibits a fractal resemblance to the platform companies themselves (Gillespie, 2018). Feudal corporate governance is most evident in the case of Facebook, where Mark Zuckerberg holds a controlling stake through a dual-class stock structure (Han, 2012). To extend the metaphor of feudalism: if admins are ladies and lords, Zuckerberg acts as a pope or monarch, who holds similarly absolutist powers over the rules by which his nobles operate, even without interfering in their fiefdoms directly. Facebook has attempted some democratic gestures, such as a 2009 


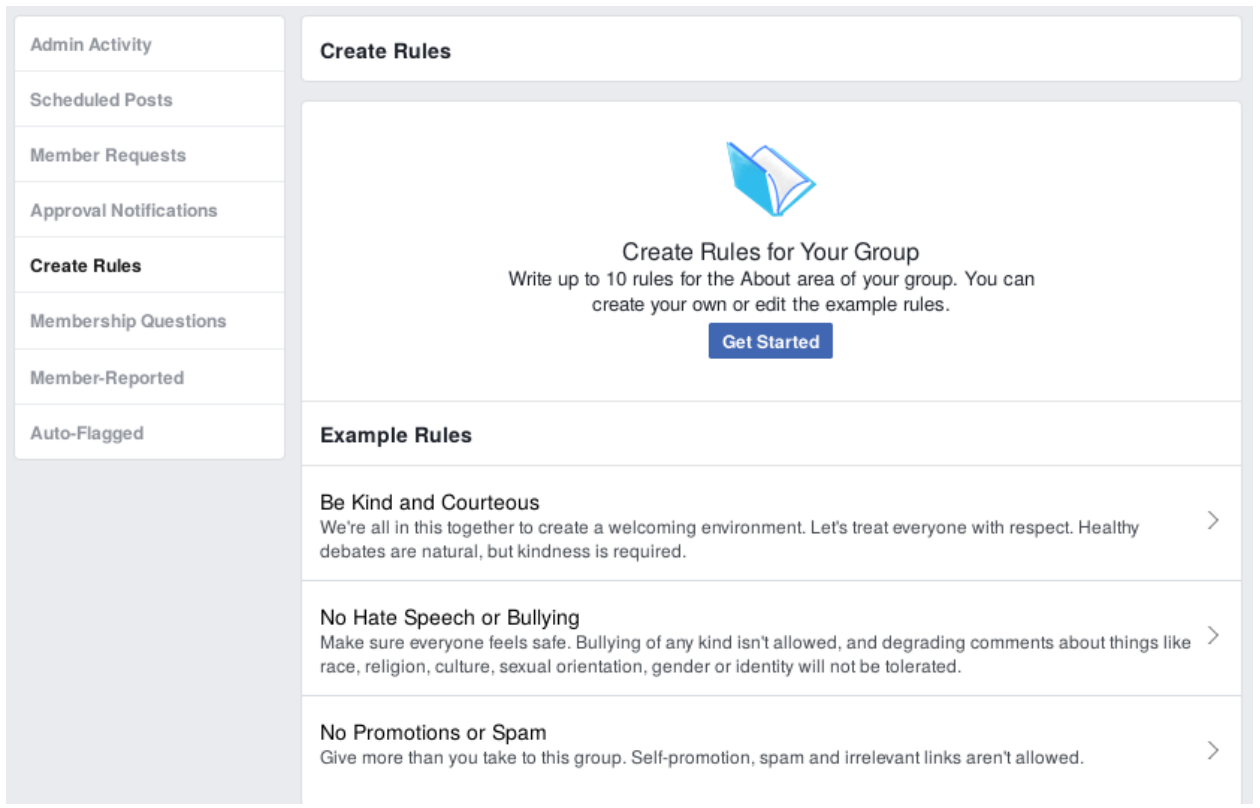

Figure 3: Rule-making interface for a Facebook group (screenshot by author).

user referendum on proposed changes to its terms of service (Bonneau et al., 2009), which fell far short of the quorum necessary to carry weight. Yet Zuckerberg rebuffs shareholder proposals to put meaningful constraints on his authority (Stewart, 2019). Reddit's corporate edifice has had its own momentary brushes with a kind of democracy, such as in the 2015 'Reddit Revolt,' when moderators protested a firing at the company by switching their Subreddits to private en masse, resulting in a widespread blackout of the platform's content and the resignation of interim CEO Ellen Pao (Matias, 2016). During the same period, however, heightened enforcement of site-wide policies brought about more conformity between the platform's policies and moderator policies at the Subreddit level (Fiesler et al., 2018).

Facebook Groups and Subreddits may represent a momentary peak for implicit feudalism. In a more recent lengthy missive, Zuckerberg (2019) pivoted from a vision of Facebook as a community-oriented 'meaningful' space to that of a 'privacy focused' platform for private chat and 'intimate' group exchanges. It was a retreat from his aspirations two years earlier for 'global community.' Already, Facebook-acquired platforms WhatsApp and Instagram were making gains against the company's namesake product. The photo-sharing app Instagram includes no functionality for groups; WhatsApp 


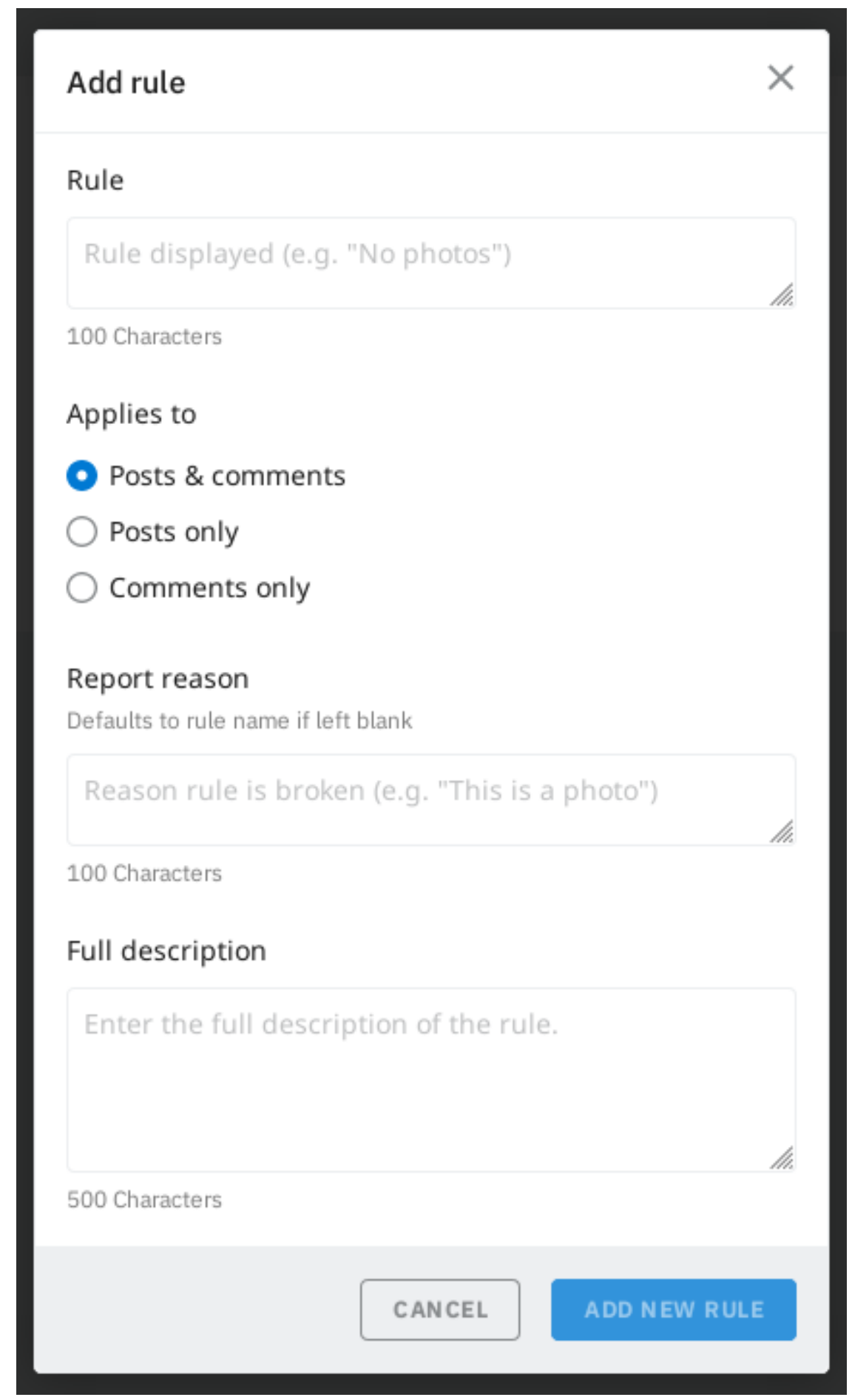

Figure 4: Rule-making interface for ${ }_{16}$ Subreddit (screenshot by author). 


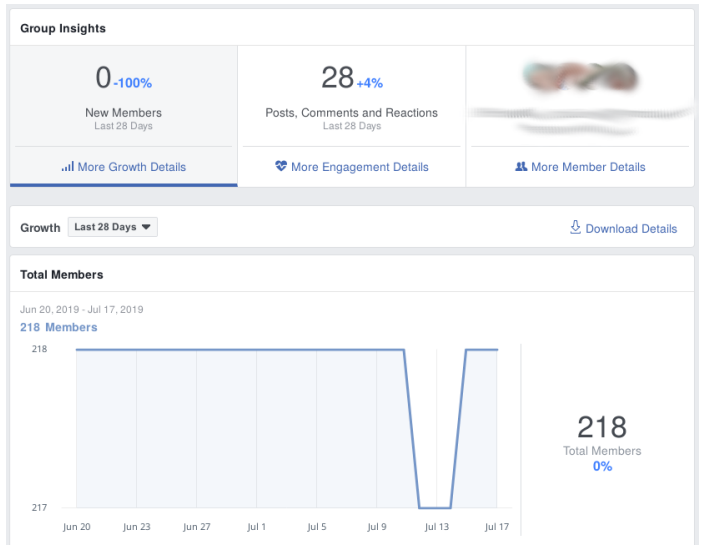

Figure 5: Analytics interface for a Facebook group (screenshot by author).

permits them only within the logic of chat, rather than Facebook's forumlike threaded discussions. Zuckerberg appears to be learning from China's WeChat in enshrining networked individuals, rather than a network of communities, as the rubric for platform society. This pivot trades feudalismwhich presumes community, however hierarchical - for platform-mediated direct relationships, apparently detached from any particular kind of politics.

\section{Feudal defaults and the possibility of voice}

Implicit feudalism has reigned over the dominant platforms for online communities so far, from the early BBSes to AI-enabled Facebook Groups. Peerproduction practices surrounding free/open-source software and Wikipedia also exhibit it. In summary, implicit feudalism's recurrent characteristics include:

- Control over communities vested in an individual or small group

- Authority derives from founders and their appointed successors

- Opacity of policy-making and decision-making processes

- Suppression of user voice as a basic privilege of authority

- User exit as the most forceful means of dissent

- Sole recourse in disputes to platform owners

While these made a specific kind of sense in the context of a BBS running in a sysop's home, that is not necessarily the case in the context of a global, multibillion-user platform like Facebook. One can just as easily imagine implicit democracy operating there as implicit feudalism. Yet feudal powers became 
part of the business model, incentivizing the unpaid labor of moderation and community building.

The feudal pattern has by and large been written into the default behaviors of online-community platforms. Exceptions like Wikipedia and Debian have required considerable, intentional effort to counteract the implicit feudalism of their tools' defaults.

Governance defaults in other domains present an instructive contrast. For instance, even quite autocratic political regimes today carry out performances of democratic institutions, such as elections and competing parties, because such practices have come to stand as prerequisites for legitimate government. Public corporations and nonprofit organizations are expected by law to be governed by boards that represent specific stakeholders - in those cases, generally shareholders and donors - together with certain transparency requirements. Even relatively informal civil-society organizations such as garden clubs and fraternal societies typically practice at least a semblance of choosing leaders by a ballot among members. Although these mechanisms of participatory governance can mask feudalism or autocracy in practice, their offline ubiquity makes it striking that no major online community software platform offers purpose-built features to support them. Adopting conventional democratic mechanisms online requires working intentionally and persistently against the grain of implicit feudalism.

The fact that implicit feudalism is so ubiquitous does not necessarily justify its ubiquity. It is certainly not universally effective for building persistent communities. The number of Subreddits with only a handful of subscribers far exceeds those that have attracted more outside users (Medvedev et al., 2019). In their study of user-run servers for the game Minecraft - a technical arrangement that resembles a BBS of old - Frey and Sumner (2019) found that the median lifetime of a server is eight weeks, and more than half of admins never recruit any committed community members. High failure rates may not be a bad thing; online communities are relatively low-risk environments, with minimal startup costs and minimal consequences of demise. But there would likely be benefits to greater institutional diversity, including in some cases an outright bias toward democratic mechanisms. Hirschman (1970) predicted that while exit-based organizational logic excels in producing variety, choice, and innovation, voice-based logic confers greater commitment and stability. In their study of the GameCenter online community, for instance, McEwan and Gutwin (2017) observed that when the platform's 'benevolent dictator' became less active over time, subgroups developed unexpected resilience. 
Platform designers can further explore techniques for enabling democracy, such as through leadership change, user representation, and shared-resource management. Platforms can also explicitly oppose feudal tendencies, as the Apache Software Foundation (2019) does for its hosted projects: 'No dictators or corporate overlords are allowed.' Following the model of offline political systems, a platform might impose obligatory elections on open groups above a certain size, through which users can campaign for positions of authority. There might be built-in term limits, campaigning rules, membership requirements, and judicial oversight. Online platforms are also poised to offer advances over offline organizations. Emerging mechanisms such as 'liquid democracy' (Hardt and Lopes, 2015) and 'holographic consensus' (Field, 2018) can facilitate direct participation across large, heterogeneous groups. As in the game League of Legends's 'tribunal' system (Kou and Nardi, 2014), an automated sortition process can form randomized juries of users to make decisions on complex questions. Blockchain-based technology has given rise to a new set of strategies for collective resource management by adopting game-theoretic reputation systems to protect against potential abuses (Reijers et al., 2018).

The more democratic regimes reviewed above, such as Debian and Wikipedia, do not exist in isolation. They act in concert with various kinds of regimes, playing distinct and complementary roles. Hirschman's exit-voice framework predicts such outcomes, as it recognizes that different institutional logics serve different purposes; they are not substitutes for each other. Simply replacing feudal governance with democratic governance anywhere and everywhere could create as many problems as it solves. Thus, rather than seeking to simply maximize the prevalence of voice-based democratic governance, designers might seek to optimize for institutional diversity (Ostrom, 2006). Such diversity might take several forms.

Debian, for instance, is not a standalone operating system; rather, it holds a particular location in a software 'stack.' The Linux kernel, with its rigidly managed dictatorship, lies beneath. Debian holds a middle space, with its democracy enabling a slow but inclusive development process that supports even older machines with limited commercial value. Above Debian sits Ubuntu, a popular operating system supported by a for-profit company, Canonical, whose founder and CEO Mark Shuttleworth uses the online handle 'sabdfl,' or 'self-appointed benevolent dictator for life.' Ubuntu benefits from the breadth of Debian but funnels that breadth into a more streamlined operating system with a faster release cycle. 
Democracy appears to flourish at certain niches in the software supply chain, while it may not be as well suited for others. Greater institutional diversity could thus allow peer-production communities to fill more kinds of roles. Democratic governance may be more likely to emerge under democratic forms of platform ownership, such as through a user-owned cooperative (Schneider, 2018). From BBSes to Facebook - or nonprofit-owned projects like like Debian and Wikipedia - community governance tends to mirror the underlying platforms' ownership structures.

Governance diversity can also unfold over time. The community that produces the Python programming language had a benevolent dictator, Guido van Rossum, for almost 30 years. When van Rossum resigned from the role in 2018, Python developers undertook a process that led to the adoption of an elected five-person 'steering council' (Edge, 2018). As in this case, a new, unproven community might be better served by feudalism, only to adopt democratic practices as it matures.

Software designers might enable greater institutional diversity by offering users a kind of 'app store' for governance. The rule-making systems already available to admins on Facebook and Reddit could be expanded to include not just behavioral rules but software-facilitated processes, such as elections, boards, juries, and petitions. Like Minecraft, platforms could have userprogrammable governance plugins, resulting in marketplaces with various modules that communities can try and adopt. These marketplaces would enable much more widespread comparative research on governance practices. They would also expose online community members to virtual worlds in which democracy is at least as available as feudalism.

\section{Conclusion: Implicit politics}

I have argued that implicit feudalism has become a nearly ubiquitous pattern embedded into the software for online communities, to the point that even apparent exceptions prove the rule. Implicitly feudal designs incline communities, like 'dark patterns,' toward the 'iron law of oligarchy.' These designs have specific, sensible historical origins but probably unnecessary persistence. Meanwhile, implicit feudalism has initiated online community members into what I have called 'imagined sufficiency' - a willingness to accept the exit logic of market fiefdoms without the voice-logic of democratic participation. To whatever extent online communities guide people's political lives and imaginations, the absence of democratic affordances may produce a de-skilling in democratic practices more generally. Implicit feudalism doesn't 
just lurk in the software; it reflects, expresses, and promulgates certain kinds of political habits.

Recognizing implicit feudalism can have explanatory virtues. As Whitney Phillips (2015) concludes, trolling and other troublesome online behaviors prompt 'a critique of dominant institutions' as much as of 'the trolls who operate within them' (p. 12); perhaps the ubiquity of feudalism in online institutions helps explain the prevalence of such disruptions. And while communication scholars have long studied voice and expression in online communities, they have shown little regard for the systematic lack of formal leverage those voices have in their own communities. Seering et al. (2019) at least suggest in passing that 'platforms might consider developing features that allow all users to get involved in self-governance if they so choose.' A growing conversation has also begun to form among scholars analyzing and designing online communities as common-pool resources, following the work of Elinor Ostrom (Frey and Sumner, 2019; Fuster Morell, 2014; Krafft et al., 2019; Kraut et al., 2011; Seidel, 2019; Silberman, 2016).

While I have sought to provide a comprehensive introduction to implicit feudalism, there is much about the concept that invites further study: 1) Future research might identify more empirically than I have the germs of democratic practice already latent in online communities, along with ways designers could encourage and amplify them. 2) Platforms might devise and test novel democratic governance techniques, even providing the means for users to develop and adopt governance mechanisms of their own. 3) Upon noticing the centrality of exit logic in online-community software, scholars and platform designers alike should rethink the ethics of relying on exit; perhaps they can then better identify conditions under which voice-based mechanisms are necessary to ensure accountability.

Once we grasp the persistence of implicit feudalism, can we continue to imagine that it is sufficient? If not, platform designers must be more intentional if they want democracy to arise in the communities they support. The inertia of implicit feudalism is strong, and by comparison online democracy has barely been tried.

\section{References}

Apache Software Foundation (2019) Incubating Issues. https://cwiki.apache.org/confluence/display/INC Benkler Y (2006) The Wealth of Networks: How Social Production Transforms 
Markets and Freedom. New Haven, CT: Yale University Press.

Big-8.org (2013) Big-8 Usenet Hierarchies. http://www.big-8.org/articles/b/i/g/Big-

8 _Usenet_hierarchies.html.

Bonneau J, Preibusch S, Anderson J, et al. (2009) Democracy Theatre: Comments on Facebook's Proposed Governance Scheme. University of Cambridge Computer Laboratory.

Caines AJ (2013) How to Run a Mailing List (Was: Please Vote Today [sic.]. Full Disclosure maling list archives. https://seclists.org/fulldisclosure/2003/Jun/396.

Castells M (2015) Networks of Outrage and Hope: Social Movements in the Internet Age. Second edition. Cambridge, UK: Polity.

Castillo O (1991) VOTEMGR: A Vote Manager for FidoNet Systems. FidoNews 8(35).

Christensen W and Seuss R (1978) Hobbyist Computerized Bulletin Board. Byte 3(11).

Clement J (2019) Global Social Media Ranking 2019. Statista. https://www.statista.com/statistics/272014/global-social-networksranked-by-number-of-users/.

Coleman EG (2013) Coding Freedom: The Ethics and Aesthetics of Hacking. Princeton: Princeton University Press.

Cosentino V, Izquierdo JLC and Cabot J (2014) Three Metrics to Explore the Openness of GitHub projects. arXiv preprint. Available at: http: //arxiv.org/abs/1409.4253.

Diamond L (2004) What is Democracy? Hilla University for Humanistic Studies.

Driscoll K (2017) Thou Shalt Love thy BBS: A Framework for the Moderation of Online Communities. In: Computer Networks Histories: Local, National and Transnational Perspectives, Università della Svizzera Italiana, Lugano, Switzerland, December 2017.

Edge J (2018) Python Gets a New Governance Model. LWN.net. https://old.lwn.net/Articles/775105/.

Evans SK, Pearce KE, Vitak J, et al. (2017) Explicating Affordances: A Conceptual Framework for Understanding Affordances in Communication 
Research. Journal of Computer-Mediated Communication 22(1): 35-52. DOI: $10.1111 /$ jcc4.12180.

Fansher M, Chivukula SS and Gray CM (2018) \#Darkpatterns: UX Practitioner Conversations About Ethical Design. In: Extended Abstracts of the 2018 CHI Conference on Human Factors in Computing Systems - CHI '18, Montreal, Canada, 2018, pp. 1-6. ACM Press. DOI: $10.1145 / 3170427.3188553$.

Federman M (2006) The Penguinist Discourse: A Critical Application of Open Source Software Project Management to Organization Development. Organization Development Journal; Chesterland 24(2): 89-100.

Field M (2018) Holographic ConsensusPart 1. DAOstack blog. https://medium.com/daostack/holographic-consensus-part-1-116a73ba1e1c.

Fiesler C, Jiang J", McCann J, et al. (2018) Reddit Rules! Characterizing an Ecosystem of Governance. In: Twelfth International AAAI Conference on Web and Social Media, June 2018.

Fish A, Murillo LFR, Nguyen L, et al. (2011) Birds of the Internet: Towards a field guide to the organization and governance of participation. Journal of Cultural Economy 4(2): 157-187. DOI: 10.1080/17530350.2011.563069.

Freeman J (1972) The Tyranny of Structurelessness. Berkeley Journal of Sociology 17: 151-164.

Frey S and Sumner RW (2019) Emergence of integrated institutions in a large population of self-governing communities. PLOS ONE 14(7): e0216335. DOI: 10.1371/journal.pone.0216335.

Fuster Morell M (2014) Governance of Online Creation Communities for the Building of Digital Commons: Viewed through the framework of the institutional analysis and development. In: Frischmann BM, Madison MJ, and Strandburg KJ (eds) Governing Knowledge Commons. New York: Oxford University Press, pp. 281-312. DOI: 10.1093/acprof:oso/9780199972036.003.0009.

Gillespie T (2018) Governance of and by Platforms. In: Burgess J, Marwick AE, and Poell T (eds) The SAGE Handbook of Social Media. 1 edition. Thousand Oaks, CA: SAGE Publications Ltd.

Hafner K and Lyon M (1996) Where Wizards Stay up Late: The Origins of the Internet. New York: Simon \& Schuster. 
Halfaker A, Geiger RS, Morgan JT, et al. (2013) The Rise and Decline of an Open Collaboration System: How Wikipedia's Reaction to Popularity Is Causing Its Decline. American Behavioral Scientist 57(5): 664-688. DOI: $10.1177 / 0002764212469365$.

Han AS (2012) The Facebook IPO's Face-off with Dual Class Stock Structure Comment. University of Michigan Journal of Law Reform Online 45: $50-55$.

Hardt S and Lopes LCR (2015) Google Votes: A Liquid Democracy Experiment on a Corporate Social Network. Technical Disclosure Commons. Defensive Publications Series.

Hirschman AO (1970) Exit, Voice, and Loyalty: Responses to Decline in Firms, Organizations, and States. Cambridge, MA: Harvard University Press.

Huhtamo E and Parikka J (2011) Media Archaeology: Approaches, Applications, and Implications. Berkeley, CA: University of California Press.

Hyman A (2003) Twenty Years of ListServ as an Academic Tool. The Internet and Higher Education 6(1): 17-24. DOI: 10.1016/S1096-7516(02)00159-8.

Jemielniak D (2014) Common Knowledge?: An Ethnography of Wikipedia. Stanford University Press.

Jhaver S, Birman I, Gilbert E, et al. (2019) Human-Machine Collaboration for Content Regulation: The Case of Reddit Automoderator. ACM Transactions on Computer-Human Interaction 26(5): 1-35. DOI: $10.1145 / 3338243$.

Kollock P and Smith M (1996) Managing the Virtual Commons: Cooperation and Conflict in Computer Communities. In: Herring SC (ed.) ComputerMediated Communication: Linguistic, Social, and Cross-Cultural Perspectives. Amsterdam: John Benjamins Publishing Company, p. 109. DOI: 10.1075/pbns.39.10kol.

Kostakis V (2010) Peer governance and Wikipedia: Identifying and understanding the problems of Wikipedia's governance. First Monday 15(3). DOI: $10.5210 /$ fm.v15i3.2613.

Kou Y and Nardi B (2014) Governance in League of Legends: A Hybrid System. In: Proceedings of the International Conference on Foundations of Digital Games, April 2014, p. 9. 
Krafft P, Keegan B and Frey S (2019) Designing for Participation and Change in Digital Institutions. arXiv:1902.08728 [cs]. Available at: http://arxiv.org/abs/1902.08728.

Kraut RE, Resnick P and Kiesler S (2011) Building Successful Online Communities: Evidence-Based Social Design. Cambridge, Mass: MIT Press.

Laffan L (2012) A New Way of Measuring Openness: The Open Governance Index. Technology Innovation Management Review 2(1): 18-24.

Leavitt A and Robinson JJ (2017) The Role of Information Visibility in Network Gatekeeping: Information Aggregation on Reddit During Crisis Events. In: Proceedings of the 2017 ACM Conference on Computer Supported Cooperative Work and Social Computing, New York, NY, USA, 2017, pp. 1246-1261. CSCW '17. ACM. DOI: 10.1145/2998181.2998299.

Ludlow P (ed.) (2001) Crypto Anarchy, Cyberstates, and Pirate Utopias. Digital communication. Cambridge, Mass: MIT Press.

Malloy J (2016) The Origins of Social Media. In: Malloy J (ed.) Social Media Archeology and Poetics. Cambridge, MA: MIT Press.

Margonelli L (1999) Inside AOL's "Cyber-Sweatshop". Wired.

Matias JN (2016) Going Dark: Social Factors in Collective Action Against Platform Operators in the Reddit Blackout. In: Proceedings of the 2016 CHI Conference on Human Factors in Computing Systems CHI '16, Santa Clara, CA, 2016, pp. 1138-1151. ACM Press. DOI: $10.1145 / 2858036.2858391$.

McEwan G and Gutwin C (2017) A Case Study of How a Reduction in Explicit Leadership Changed an Online Game Community. Computer Supported Cooperative Work (CSCW) 26(4): 873-925. DOI: 10.1007/s10606-0179282-0.

Medvedev AN, Lambiotte R and Delvenne J-C (2019) The Anatomy of Reddit: An Overview of Academic Research. In: Dynamics On and Of Complex Networks III (eds F Ghanbarnejad, R Saha Roy, F Karimi, et al.), 2019, pp. 183-204. Springer Proceedings in Complexity. Springer International Publishing.

Nagy P and Neff G (2015) Imagined Affordance: Reconstructing a Keyword for Communication Theory. Social Media + Society 1(2). DOI: $10.1177 / 2056305115603385$. 
Noble SU (2018) Algorithms of Oppression: How Search Engines Reinforce Racism. New York: NYU Press.

OneNet (1999) The OneNet Member Constitution. Wayback Machine Archive. https://web.archive.org/web/19980623215303fw_/http://www.onenet.org/Cons/Intro.htm.

Ostrom E (2006) Understanding Institutional Diversity. Princeton, NJ: Princeton University Press.

Phillips W (2015) This Is Why We Can't Have Nice Things: Mapping the Relationship Between Online Trolling and Mainstream Culture. Cambridge, MA: MIT Press.

Reagle JM (2010) Good Faith Collaboration: The Culture of Wikipedia. Cambridge, MA: MIT Press.

Reijers W, Wuisman I, Mannan M, et al. (2018) Now the Code Runs Itself: On-Chain and Off-Chain Governance of Blockchain Technologies. Topoi. DOI: $10.1007 / \mathrm{s} 11245-018-9626-5$.

Rheingold H (1993) The Virtual Community: Homesteading on the Electronic Frontier. Reading, MA: Addison-Wesley.

Schneider N (2018) An Internet of Ownership: Democratic Design for the Online Economy. The Sociological Review 66(2).

Schneider N (2019) Decentralization: An Incomplete Ambition. Journal of Cultural Economy 12(4): 1-21. DOI: 10.1080/17530350.2019.1589553.

Schrock AR (2015) Communicative Affordances of Mobile Media: Portability, Availability, Locatability, and Multimediality.: 18.

Scott J (2005) BBS: The Documentary. Bovine Ignition Systems.

Seering J, Wang T, Yoon J, et al. (2019) Moderator engagement and community development in the age of algorithms. New Media 85 Society. DOI: $10.1177 / 1461444818821316$.

Seidel N (2019) Democratic Power Structures in Virtual Communities. In: Proceedings of the 24th European Conference on Pattern Languages of Programs, New York, NY, USA, 2019, pp. 31:1-31:8. EuroPLop '19. ACM. DOI: 10.1145/3361149.3361181.

Shaw A and Hill BM (2014) Laboratories of Oligarchy? How the Iron Law Extends to Peer Production. Journal of Communication 64(2): 215-238. DOI: $10.1111 /$ jcom.12082. 
Silberman MS (2016) Reading Elinor Ostrom In Silicon Valley: Exploring Institutional Diversity on the Internet. In: Proceedings of the 19th International Conference on Supporting Group Work, New York, 2016, pp. 363-368. GROUP '16. ACM. DOI: 10.1145/2957276.2957311.

Spencer H and Lawrence D (1998) Managing Usenet. Sebastopol, CA, USA: O'Reilly \& Associates.

Stasavage D (2020) The Decline and Rise of Democracy: A Global History from Antiquity to Today. Princeton: Princeton University Press.

Stewart E (2019) Facebook Will Never Strip Away Mark Zuckerberg's Power. Vox. https://www.vox.com/recode/2019/5/30/18644755/facebook-stockshareholder-meeting-mark-zuckerberg-vote.

Tourani P, Adams B and Serebrenik A (2017) Code of conduct in open source projects. In: 2017 IEEE 24th International Conference on Software Analysis, Evolution and Reengineering (SANER), February 2017, pp. 24-33. DOI: 10.1109/SANER.2017.7884606.

Tufekci Z (2017) Twitter and Tear Gas: The Power and Fragility of Networked Protest. New Haven, CT: Yale University Press.

Wikipedia (2020) Wikipedia:Neutral point of view. Wikipedia.

Zacchiroli S (2011) Debian: 18 Years of Free Software, Do-ocracy, and Democracy. In: Proceedings of the 2011 Workshop on Open Source and Design of Communication - OSDOC '11, Lisbon, Portugal, 2011, p. 87. ACM Press. DOI: 10.1145/2016716.2016740.

Zuboff S (2019) The Age of Surveillance Capitalism: The Fight for a Human Future at the New Frontier of Power. New York: PublicAffairs.

Zuckerberg M (2017) Building Global Community. Facebook. https://www.facebook.com/notes/markzuckerberg/building-global-community/10154544292806634.

Zuckerberg M (2019) A Privacy-Focused Vision for Social Networking. Facebook. https://www.facebook.com/notes/mark-zuckerberg/a-privacyfocused-vision-for-social-networking/10156700570096634/. 